\title{
Drug discovery is an eternal challenge for the biomedical sciences
}

\author{
Hua Li, ${ }^{a}$ Wenyi Wei ${ }^{b, *}$ and Hongxi $X^{c, *}$ \\ aWuya College of Innovation, Key Laboratory of Structure-Based Drug Design \& Discovery, Ministry of Education, Shenyang Pharmaceutical \\ University, Shenyang, China \\ School of Pharmacy, Tongji Medical College, Huazhong University of Science and Technology, Wuhan, China \\ bepartment of Pathology, Beth Israel Deaconess Medical Center, Harvard Medical School, Boston, MA 02215, United States \\ 'School of Pharmacy, Shanghai University of Traditional Chinese Medicine, Shanghai 201203, China
}

*Correspondence: wwei2@bidmc.harvard.edu (W.Wei); xuhongxi88@gmail.com (H. Xu)

Published online: 01 January 2022

DOI 10.15212/AMM-2022-1001

Throughout history, humans have dreamed of finding a panacea for diseases and an elixir for immortality. As alluded to in the popular Chinese legend of Shennong, who tasted hundreds of herbs in a day to test their medical value, for thousands of years before the emergence of modern medicinal science, only natural materia medica and medication methods derived primarily from experience could be used to treat diseases. When epidemics wreak havoc and medicine cannot provide relief, witchcraft prevails but eventually fails to overcome the disease. Various lethal human diseases, such as plague, smallpox and tuberculosis, persisted and claimed countless human lives throughout history until they were finally harnessed by the power of medical sciences.

With the development of modern medical sciences based on anatomy, physiology, microbiology and immunology, drug discovery is no longer a merely accidental process depending solely on experience. The rise of the modern chemical and pharmaceutical industry has enabled the use of not only natural sources but also chemically synthesized substances to combat various forms of human diseases. For example, the application of sulfonamide, penicillin and streptomycin antibiotics temporarily relieved humans of the nightmare of bacterial infectious diseases.

Driven by the discovery of the double-helix structure of DNA and the development of molecular biology, the understanding of diseases and drug targets has deepened, extending to the genetic and molecular levels. Moreover, owing to the development of protein structural biology, direct interactions between small drug molecules and their target macromolecules can be captured. These scientific advances led to the discovery of the first targeted antitumor drug, imatinib, for the treatment of chronic myeloid leukemia [1].

Despite the emergence of chemically synthesized drugs, natural products remain the basis and major resource for drug innovation. Currently, half the drugs on the market are derived from natural products and related molecules [2]. Among them, artemisinin, derived from traditional Chinese medicine, is a notable example [3]. The discovery of natural product lead compounds and mechanisms of action has been, and will continue to be, the frontier of fundamental research and a promising path to the discovery of new drug targets and cellular signaling pathways [4].

It is also worth noting that progress in the resolution limit of complex structures determined by threedimensional reconstruction technology of Cryo-EM at a near- atomic level renders it a new and powerful tool for drug discovery $[5,6]$. The growing number of successful determinations of drug-target co-structure by cryo-EM makes us hopeful that this technique will keep pace with protein crystallography in the field of drug discovery in the near future $[7,8]$.

Beyond experimental methods, the success of Alphafold-2 has enabled the prediction of accurate three-dimensional structures of drug targets on a large scale [9]. The rapid development of artificial intelligence has positioned information technology to play an increasingly important role in the preclinical stage of drug discovery, particularly in virtual ligand screening, rational drug design and the prediction of pharmacokinetic and toxicological characteristics, thus allowing candidate drugs to be obtained with reduced cost and time [10]. This important cornerstone of drug discovery marks its shift from the realm of chance to the realm of necessity. The recent success of Pfizer's anti-COVID-19 drug, Paxlovid, perfectly illustrates both the strength and necessity of rational drug design [11].

According to data released by the WHO, infectious diseases caused by bacteria, viruses and parasites are leading causes of human death, accounting for approximately $25 \%$ of all deaths due to disease [12]. On the one 
hand, antibiotics, once regarded as the pinnacle of medicine and an effective weapon against bacterial infections, have gradually lost their advantages partly because of the emergence of multidrug-resistant and extensively drug-resistant strains [13, 14]. Secondary infection of drug-resistant bacteria, such as multidrug-resistant Mycobacterium tuberculosis, Staphylococcus aureus and other bacterial infections in hospitals are becoming major threats to global health. Super-bacteria that are resistant to almost all antibiotics have also emerged [15, 16]. The rate of development of new antibiotics has lagged far behind the rate of the emergence of new bacterial drug resistance. On the other hand, since the 21st century, new viral infections such as H1N1 influenza, Ebola, Zika, poliomyelitis and COVID-19 have caused several Public Health Emergencies of International Concern [17], particularly coronavirus infections, which have caused several fatal human pneumonia outbreaks, including severe acute respiratory syndrome [18], Middle East respiratory syndrome [19] and the currently ongoing outbreak of COVID-19 caused by the SARS-CoV-2 virus [20]. New and recurrent zoonoses have remained a major threat to worldwide public health [21]. Therefore, in the postCOVID-19 era, the development of anti-drug-resistant bacteria and antiviral drugs will continue to be main themes in drug discovery.

Another situation that cannot be ignored is that many countries worldwide, particularly developed countries have increasingly aging populations. Chronic diseases associated with aging, such as cancer, cardiovascular and cerebrovascular diseases, neurodegenerative diseases and metabolic diseases have become major health problems in these countries [12]. The medical expenditure for the treatment of these diseases accounts for an increasing proportion of governmental and insurance company budgets. Therefore, in the coming decades, the main efforts in drug discovery and development are expected to remain focused on fields associated with these chronic diseases. In addition, drug research and development in the field of anti-aging is also expected to experience a rapid boom.

Since the beginning of the 21st century, with the completion of the human genome project [22], biomedical sciences have entered the postgenome era, and new tools and concepts have proliferated. The development of fundamental biomedical research itself has promoted the discovery of innovative drugs. For example, breakthroughs in nucleic acid research have made mRNA vaccines and gene-editing therapies possible [23, 24]; the study of tumor immune escape has led to PD-1/PD-L1 antibody therapy and CAR-T cell therapy [25, 26]; and the study of ubiquitination-degradation systems and the autophagy system have enabled protein-targeted-degradation drugs (e.g., protacs, lytacs, autacs and attecs) [27-30]. Moreover, the study of small drug molecules as probes can sometimes work in reverse by promoting fundamental research. For instance, the study of rapamycin as a molecular glue resulted in intensive promotion of fundamental research in the field of antiaging [31].

In this era in which the rate of advancement of the theoretical underpinnings of basic disciplines such as physics and mathematics has slowed, biomedical sciences have risen to become the prominent revolutionary force of scientific development. The pursuit of health and immortality powers drug discovery. Countless innovative concepts and technologies as diverse as molecular glue [32], bispecific antibodies [33], protacs [27], antibody-drug conjugates [34], DNA-encoded libraries [35], allosteric regulators [36], multiomics technologies [37] smart drug-delivery systems [38] and synthetic biology of natural products [39], indicate that we are in a challenging but promising era of change. We must listen to the demands of patients, doctors and regulatory authorities, as well as the voices of science, conscience and humanity. The ultimate goal of drug discovery is to produce a panacea to treat diseases and enhance the health and happiness of all humanity. To progress toward this yet-distant goal, we call for cooperation, mutual compromise and joint efforts among scientists, investors, industrial manufacturers and policymakers. In the most optimistic scenario, drugs should be created but eventually will no longer be needed.

The discovery of new drugs is among humanity's most sophisticated and cutting-edge intellectual activities. This process requires not only the discovery of the basic laws governing biological activities with curiosity, but also the inspirational creation of new materials, and decades of persistence in the face of difficulties and failures. This pursuit is both interdisciplinary and comprehensive, and such a grand project could not be advanced without the large-scale collaboration of numerous talented minds. Non est ad astra mollis e terris via: there is no easy way from the earth to the stars.

\section{ACKNOWLEDGMENTS}

We thank Kexing Li from the School of Life Sciences, Tsinghua University, for English editing help.

\section{CONFLICTS OF INTEREST}

Hua Li is Executive Editor of this journal. Wenyi Wei and Hongxi $\mathrm{Xu}$ are Co-Editors in Chief of this journal.

\section{REFERENCES}

[1] Buchdunger E, Zimmermann J, Mett H, Meyer T, Müller $\mathrm{M}$, Druker BJ, et al.: Inhibition of the Abl Protein-Tyrosine Kinase In Vitro and In Vivo by a 2-Phenylaminopyrimidine Derivative. Cancer Research 1996, 56(1):100-104.

[2] Kakeya H: Natural Products-Prompted Chemical Biology: Phenotypic Screening and a New Platform for Target Identification. Natural Product Reports 2016, 33(5):648-654. 
[3] Kong LY, Tan RX: Artemisinin, a Miracle of Traditional Chinese Medicine. Natural Product Reports 2015, 32(12):1617-1621.

[4] Harvey AL, Edrada-Ebel R, Quinn RJ: The Re-Emergence of Natural Products for Drug Discovery in the Genomics Era. Nature Reviews Drug Discovery 2015, 14(2):111-129.

[5] Lees JA, Dias JM, Han S: Applications of Cryo-EM in Small Molecule and Biologics Drug Design. Biochemical Society Transactions 2021, BST20210444.

[6] de Oliveira TM, van Beek L, Shilliday F, Debreczeni JÉ, Phillips C: Cryo-EM: The Resolution Revolution and Drug Discovery. SLAS Discovery 2021, 26(1):17-31.

[7] Wang $N$, Jiang $X$, Zhang $S$, Zhu A, Yuan $Y, X u ~ H$, et al.: Structural Basis of Human Monocarboxylate Transporter 1 Inhibition by Anti-Cancer Drug Candidates. Cell 2021, 184(2):370-383.e13.

[8] Van Drie JH, Tong L: Cryo-EM as a Powerful Tool for Drug Discovery. Bioorganic \& Medicinal Chemistry Letters 2020, 30(22):127524.

[9] Tunyasuvunakool K, Adler J, Wu Z, Green T, Zielinski M, Žídek A, et al.: Highly Accurate Protein Structure Prediction for the Human Proteome. Nature 2021, 596(7873): 590-596.

[10] Vijayan RSK, Kihlberg J, Cross JB, Poongavanam V: Enhancing Preclinical Drug Discovery with Artificial Intelligence. Drug Discovery Today 2021, S1359-6446(21):00504-3.

[11] Owen DR, Allerton CMN, Anderson AS, Aschenbrenner $L$, Avery M, Berritt S, et al. An oral SARSCoV-2 Mpro inhibitor clinical candidate for the treatment of COVID-19. Science 2021, 374(6575):1586-1593.

[12] [https://www.who.int/data/global-health-estimates/]

[13] Horcajada JP, Montero M, Oliver A, Sorlí L, Luque S, Gómez-Zorrilla $S$, et al.: Epidemiology and Treatment of Multidrug-Resistant and Extensively Drug-Resistant Pseudomonas Aeruginosa Infections. Clinical Microbiology Reviews 2019, 32(4):e00031-19.

[14] El-Sayed Ahmed MAE, Zhong LL, Shen C, Yang Y, Doi Y, Tian GB: Colistin and its Role in the Era of Antibiotic Resistance: An Extended Review (2000-2019). Emerging Microbes \& Infections 2020, 9(1):868-885.

[15] Ibrahim S, Al-Saryi N, Al-Kadmy IMS, Aziz SN: MultidrugResistant Acinetobacter Baumannii as an Emerging Concern in Hospitals. Molecular Biology Reports 2021, 48(10):6987-6998.

[16] Shah I, Poojari V, Meshram H: Multi-Drug Resistant and Extensively-Drug Resistant Tuberculosis. Indian Journal of Pediatrics 2020, 87(10):833-839.

[17] Wilder-Smith A, Osman S: Public Health Emergencies of International Concern: A Historic Overview. Journal of Travel Medicine 2020, 27(8):taaa227.

[18] Holmes KV: SARS Coronavirus: A New Challenge for Prevention and Therapy. Journal of Clinical Investigation 2003, 111(11):1605-1609.

[19] Lu L, Liu Q, Du L, Jiang S: Middle East Respiratory Syndrome Coronavirus (MERS-CoV): Challenges in Identifying its Source and Controlling its Spread. Microbes and Infection 2013, 15(8-9):625-629.

[20] Zhu N, Zhang D, Wang W, Li X, Yang B, Song J, et al.: A Novel Coronavirus from Patients with Pneumonia in China, 2019. The New England Journal of Medicine 2020, 382(8):727-733.
[21] Dong X, Soong L: Emerging and Re-Emerging Zoonoses are Major and Global Challenges for Public Health. Zoonoses 2021, 1(1).

[22] Lander ES, Linton LM, Birren B, Nusbaum C, Zody MC, Baldwin J, et al.: Initial Sequencing and Analysis of the Human Genome. Nature 2001, 409(6822):860-921.

[23] Huang Q, Zeng J, Yan J: COVID-19 mRNA Vaccines. Journal of Genetics and Genomics 2021, 48(2):107-114.

[24] Sharma G, Sharma AR, Bhattacharya M, Lee SS, Chakraborty C: CRISPR-Cas9: A Preclinical and Clinical Perspective for the Treatment of Human Diseases. Molecular Therapy 2021, 29(2):571-586.

[25] Dong H, Strome SE, Salomao DR, Tamura H, Hirano F, Flies DB, et al.: Tumor-Associated B7-H1 Promotes T-Cell Apoptosis: A Potential Mechanism of Immune evasion. Nature Medicine 2002, 8(8):793-800.

[26] Sterner RC, Sterner RM: CAR-T Cell Therapy: Current Limitations and Potential Strategies. Blood Cancer Journal 2021, 11(4):69.

[27] Sakamoto KM, Kim KB, Kumagai A, Mercurio F, Crews CM, Deshaies RJ: Protacs: Chimeric Molecules that Target Proteins to the Skp1-Cullin-F Box Complex for Ubiquitination and Degradation. Proceedings of the National Academy of Sciences of the United States of America 2001, 98(15):8554-8559.

[28] Banik SM, Pedram K, Wisnovsky S, Ahn G, Riley NM, Bertozzi CR: Lysosome-Targeting Chimaeras for Degradation of Extracellular Proteins. Nature 2020, 584(7820):291-297.

[29] Takahashi D, Moriyama J, Nakamura T, Miki E, Takahashi E, Sato A, et al.: AUTACs: Cargo-Specific Degraders Using Selective Autophagy. Molecular Cell 2019, 76(5):797-810. e10.

[30] Li Z, Wang C, Wang Z, Zhu C, Li J, Sha T, et al.: AlleleSelective Lowering of Mutant HTT Protein by HTT-LC3 Linker Compounds. Nature 2019, 575(7781):203-209.

[31] Schreiber SL: Immunophilin-Sensitive Protein Phosphatase Action in Cell Signaling Pathways. Cell 1992, 70(3):365-368.

[32] Schreiber SL: The Rise of Molecular Glues. Cell 2021, 184(1):3-9.

[33] Kontermann RE, Brinkmann U: Bispecific Antibodies. Drug Discovery Today 2015, 20(7):838-847.

[34] Diamantis N, Banerji U: Antibody-Drug Conjugates - An Emerging Class of Cancer Treatment. British Journal of Cancer 2016, 114(4):362-367.

[35] Mannocci L, Leimbacher $M$, Wichert $M$, Scheuermann J, Neri D: 20 Years of DNA-Encoded Chemical Libraries. Chemical Communications 2011, 47(48):12747-12753.

[36] Lu S, Shen Q, Zhang J: Allosteric Methods and Their Applications: Facilitating the Discovery of Allosteric Drugs and the Investigation of Allosteric Mechanisms. Accounts of Chemical Research 2019, 52(2):492-500.

[37] Mardinoglu A, Boren J, Smith U, Uhlen M, Nielsen J: Systems Biology in Hepatology: Approaches and Applications. Nature Reviews Gastroenterology \& Hepatology 2018, 15(6):365-377.

[38] Jia L, Zhang $P$, Sun $H$, Dai $Y$, Liang $S$, Bai $X$, et al.: Optimization of Nanoparticles for Smart Drug Delivery: A Review. Nanomaterials 2021, 11(11):2790.

[39] Breitling R, Takano E: Synthetic Biology of Natural Products. Cold Spring Harbor Perspectives in Biology 2016, 8(10):a023994. 